\title{
Measurement and design of refractive corrections using ultrafast laser-induced intra-tissue refractive index shaping in live cats
}

Daniel R. Brooks, Kaitlin T. Wozniak, Wayne Knox, Jonathan D. Ellis, Krystel R. Huxlin

Daniel R. Brooks, Kaitlin T. Wozniak, Wayne Knox, Jonathan D. Ellis, Krystel R. Huxlin, "Measurement and design of refractive corrections using ultrafast laser-induced intra-tissue refractive index shaping in live cats," Proc. SPIE 10474, Ophthalmic Technologies XXVIII, 104741B (8 March 2018); doi: $10.1117 / 12.2290132$

SPIE. Event: SPIE BiOS, 2018, San Francisco, California, United States 


\title{
Measurement and design of refractive corrections using ultrafast laser- induced intra-tissue refractive index shaping in live cats
}

\author{
Daniel R. Brooks*a, Kaitlin T. Wozniak ${ }^{\mathrm{a}}$, Wayne Knox ${ }^{\mathrm{a}, \mathrm{c}}$, Jonathan D. Ellis ${ }^{\mathrm{a}, \mathrm{b}, \mathrm{d}}$, Krystel R. Huxlin ${ }^{\mathrm{c}}$,
} ${ }^{a}$ The Institute of Optics, University of Rochester, Rochester, NY 14611; ${ }^{b}$ Department of Mechanical Engineering, University of Rochester, Rochester, NY 14611 ; ${ }^{\mathrm{C}}$ Flaum Eye Institute, University of Rochester Medical Center, Rochester, NY 14611; ${ }^{\mathrm{d} C o l l e g e}$ of Optics Sciences, University of Arizona, 1630 E University Blvd, Tucson, AZ 85719

\begin{abstract}
Intra-Tissue Refractive Index Shaping (IRIS) uses a $405 \mathrm{~nm}$ femtosecond laser focused into the stromal region of the cornea to induce a local refractive index change through multiphoton absorption. This refractive index change can be tailored through scanning of the focal region and variations in laser power to create refractive structures, such as gradient index lenses for visual refractive correction. Previously, IRIS was used to create $2.5 \mathrm{~mm}$ wide, square, $-1 \mathrm{D}$ cylindrical refractive structures in living cats. In the present work, we first wrote $400 \mu \mathrm{m}$ wide bars of refractive index change at varying powers in enucleated cat globes using a custom flexure-based scanning system. The cornea and surrounding sclera were then removed and mounted into a wet cell. The induced optical phase change was measured with a MachZehnder Interferometer (MZI), and appeared as fringe displacement, whose magnitude was proportional to the refractive index change. The interferograms produced by the MZI were analyzed with a Fourier Transform based algorithm in order to extract the phase change. This provided a phase change versus laser power calibration, which was then used to design the scanning and laser power distribution required to create $-1.5 \mathrm{D}$ cylindrical Fresnel lenses in cat cornea covering an area $6 \mathrm{~mm}$ in diameter. This prescription was inscribed into the corneas of one eye each of two living cats, under surgical anesthesia. It was then verified in vivo by contrasting wavefront aberration measurements collected preIRIS with those obtained over six months post-IRIS using a Shack-Hartmann wavefront sensor.
\end{abstract}

Keywords: Vision correction, Multiphoton, Laser Refractive Correction, Gradient Index, Femtosecond, Cornea

\section{INTRODUCTION}

Ever since it was first shown in 1983 that a laser could be used to perform surgery on an eye ${ }^{1}$, lasers have increasingly been implemented in the realm of refractive correction. This first surgery and the procedures developed over the following years used the ultraviolet (UV) light emitted by excimer lasers to ablate the stroma of the cornea to alter the curvature of the corneal surface, thereby changing the refractive power of the eye. ${ }^{2}$ Later, the procedure called photorefractive keratectomy (PRK) was developed in 1988 by Robert Munnerlyn. ${ }^{3}$ Building on PRK, laser in situ keratomileusis (LASIK) was developed in 1990 by Pallikaris. ${ }^{4}$ While both procedures change corneal shape by removing material, PRK and LASIK differ in how the corneal stroma is exposed for laser ablation. In PRK, the epithelium, the outer most protective layer of the eye, is debrided, revealing the outermost layer of the stroma. In LASIK, an incision is made to create a flap containing the epithelium and outer layer of the stroma, which is then folded back to expose the mid-stroma of the cornea. Both of these methods can result in undesirable side effects from the removal of material and the cutting or removing of the epithelium.,

In 2006, Ding et al. first demonstrated that large refractive index (RI) changes, as high as 0.06 , could be induced in hydrogel polymers, such as those used in contact lenses and intraocular lenses, using a high repetition rate femtosecond laser (93 MHz). However, the writing speed was extremely slow, only on the order of $0.4 \mu \mathrm{m} / \mathrm{s}^{7}$ By doping with dyes which enhanced the multi-photon absorption of laser light, it was discovered that this scanning speed could be increased significantly, inducing similar levels of RI change at speeds of millimeters per second. ${ }^{8} \mathrm{Xu}$ and colleagues built on this work to create lateral gradient index (GRIN) lenses in hydrogel samples, whereby the translation speed of the focal region was varied across the lens area, thus creating a spatially varying RI profile that enabled focusing of light. $^{9}$

Ophthalmic Technologies XXVIII, edited by Fabrice Manns, Per G. Söderberg, Arthur Ho, Proc. of SPIE

Vol. 10474, 104741B · C 2018 SPIE · CCC code: 1605-7422/18/\$18 · doi: 10.1117/12.2290132 
In 2008, it was first shown that laser-induced RI change could be achieved in cornea, where it was termed intratissue refractive index shaping (IRIS). ${ }^{10}$ The first GRIN lines inscribed using IRIS were created using a $93 \mathrm{MHz}$ Ti:Sapphire oscillator operating at $800 \mathrm{~nm}$. This laser produced $0.3 \mathrm{~nJ}$ pulses, operating well below the damage threshold of the cornea. RI changes of 0.008 were observed with a scanning speed of $0.7 \mu \mathrm{m} / \mathrm{s} .{ }^{10,11}$ If, before writing, the cornea is doped with sodium fluorescein, which has a large two photon cross-section at $800 \mathrm{~nm}$, significantly larger changes in RI can be achieved at significantly higher scanning speeds. The observed RI change was up to 0.015 at a scan speed of 5 $\mathrm{mm} / \mathrm{s}^{12,13}$

While material doping represented a large step forward, applying dopants to the cornea requires that the epithelium be scraped away. This, in turn, replicates many of the problems involved in PRK. One solution to eliminate the need for doping was to frequency-double the Ti:Sapphire laser to $400 \mathrm{~nm}$. The cornea has significant native twophoton absorption at $400 \mathrm{~nm}$, which allowed RI change as high as 0.037 to be attained at $5 \mathrm{~mm} / \mathrm{s}$, and RI change of 0.021 to be achieved at $15 \mathrm{~mm} / \mathrm{s}^{14}$ After developing a new system for performing IRIS in vivo ${ }^{15},-1 \mathrm{D}$ GRIN cylindrical structures were written in living cats for the first time. ${ }^{16}$ Due to limitations of the original in vivo system, these first structures were small, $2.5 \mathrm{~mm}$ by $2.5 \mathrm{~mm}$ squares. In addition to the intended cylinder power, a significant amount of defocus was induced in these structures. The present work represents a significant advance relative to these original experiments, moving toward improved metrology and sufficiently large structures to cover the entire clinically-relevant optical zone of the human eye.

\section{CALIBRATION OF PHASE CHANGE IN ENUCLEATED CAT GLOBES}

\subsection{IRIS system}

The IRIS writing system used for the work presented in this manuscript utilized a frequency doubled Ti:Sapphire laser, operating at a wavelength of $405 \mathrm{~nm}$, to create bulk optical phase change directly in the cornea. This laser was directed through an acousto-optic modulator (AOM) in order to enable in process, fast laser power control. The AOM uses an acoustic wave to diffract a portion of the laser light into the $1^{\text {st }}$ diffracted order. The amount of diffracted light is dependent on the amplitude of the acoustic wave. The $0^{\text {th }}$ order undiffracted light was blocked by an iris and the $1^{\text {st }}$ order light was used for the remainder of the system. The light then passed through a pair of F2 prisms to compensate for dispersion, producing a final pulse width of 165 fs. Following this, the beam was directed through a beam expander in order to enlarge the beam size at the entrance pupil of a microscope objective, thus increasing the effective NA. Due to thermal bloom in the prisms, the NA was laser power dependent and ranged from 0.55 at higher powers to 0.7 at lower powers. Finally, the beam entered a scanning system designed around a custom flexure-based stage ${ }^{15}$, which scanned the focal region of an attached water immersion objective through the cornea. The flexure stage is driven with four voice coil (VC) motors. A raster motion is achieved with a linear stage stepping orthogonal to the flexure stage motion in sync with the flexure-based stage turnarounds, resulting in a dense pattern of parallel lines of local phase change. Light passing through these dense line patterns sees a bulk phase change, which is the value measured and used in the remainder of this work. The flexure-based stage is mounted on a lift stage for vertical position control to set the depth of the IRIS structure and create multiple layers of phase change. A diagram of the IRIS writing system is shown in Fig 1. 

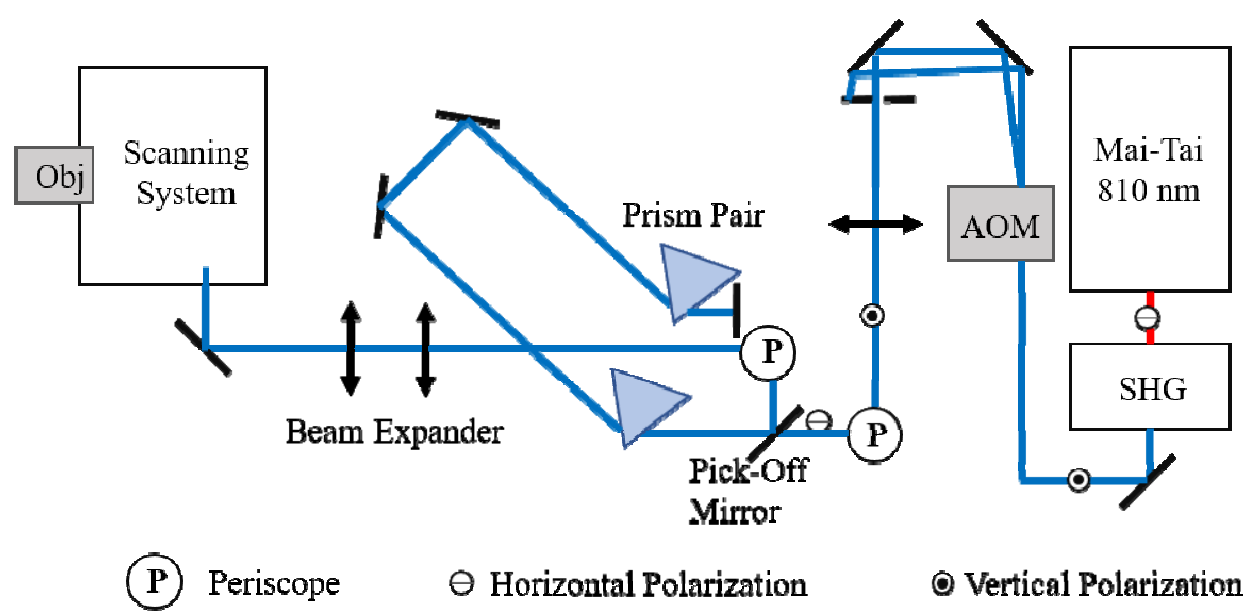

O Vertical Polarization

Figure 1: IRIS writing system. $810 \mathrm{~nm}$ Ti:Sapphire laser emission is frequency doubled to $405 \mathrm{~nm}$. Light is directed through an AOM and the $1^{\text {st }}$ order diffracted beam is picked off to be directed through the rest of the system. This passes through a dispersion compensating prism pair and a beam expander before being directed into the custom flexure-based scanning system which is used to scan the focal spot of the laser through a sample, creating large area IRIS patterns

\subsection{Phase Calibration Procedure}

The first step towards writing deterministic refractive structures in live cats is metrology of the bulk optical phase change with respect to the delivered laser power. For this work, scanning speed profile was held constant to simplify the calibration. Laser power was more easily changed in-process. A calibration function was found by writing a series of bars of constant induced phase change, each at different laser powers with laser power constant across each individual bar, in whole, enucleated cat globes. These globes were obtained from Liberty Research, Inc., Waverly, NY They were shipped overnight, on ice, and the phase bars were written the next day. Between their removal from the animal and the writing of the phase bars, the eyes were stored in Optisol-GS (Bausch \& Lomb, Inc.) and kept on ice for transportation, then refrigerated until used in the experiment.

In preparation for writing the phase bars, the eyes were placed in conical depressions in a block of plastic, partially submerged in Optisol-GS, with the corneas oriented facing up. An applanator, shown in Figure 2, was placed on the eye and vacuum was applied with a spring-loaded syringe. Once the cornea was suctioned to the applanator, the surface of the coverslip was found using a back-reflection monitor (BRM) composed of a singlet lens with a CCD camera located at the focal plane. The singlet lens collected the small amount of laser light reflected back through the objective, imaging it onto the CCD. When the focus of the objective is close to a surface, the image of the reflection approaches a small dot on the CCD. The surface was found by moving the objective vertically to adjust the distance between the objective and the applanator until the size of the image of the reflection from the coverslip-cornea interface was minimized. The surface was found at two points on either side of the applanated zone. As long as these points were within approximately $50 \mu \mathrm{m}$ of each other, signifying that the objective plane of motion and the coverslip were tolerably parallel, the locations were averaged and the average used to indicate the corneal surface. 


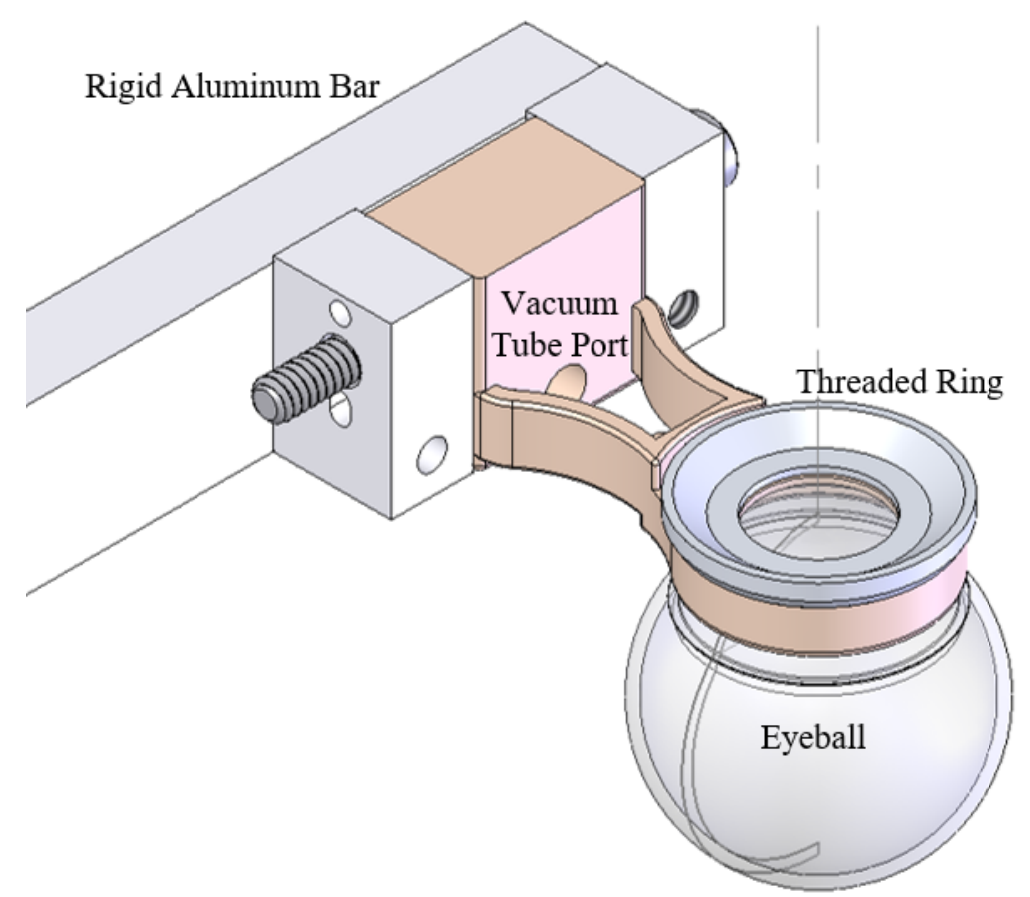

Figure 2: CAD drawing of the applanator incorporating the curved eyeball interface, threaded ring clamp for the coverslip, and rigid aluminum bar for mounting to the optical table.

Once the surface of the cornea was identified, the objective was moved to the starting location at one end of the set of phase bars and the bars were written. The parameters used for the calibration phase bars are given in Table 1. Figure 3 shows a diagram of the calibration bar geometry and a picture of an eyeball immediately after writing. In addition to the parameters in Table 1, which were held constant, the laser power was varied bar to bar to create a phase change calibration with respect to delivered laser power, which could then be converted to a calibration with respect to AOM voltage before writing the final, refractive structures.

Table 1: Writing Parameters for Phase Change Calibration

\begin{tabular}{c|c} 
Parameter & Value \\
\hline Bar width & $0.4 \mathrm{~mm}$ \\
Bar Spacing & $0.6 \mathrm{~mm}$ \\
Bar Length (measured) & $6.5 \mathrm{~mm}$ \\
Line Spacing & $0.5 \mu \mathrm{m}$ \\
VC Drive Frequency & $10 \mathrm{~Hz}$ \\
VC Drive Voltage & $1.5 \mathrm{~V}$ \\
Beam Expansion Ratio & $1: 4$ \\
Pulse Width & $169 \mathrm{fs}$ \\
\# of Layers & 3 \\
Layer Spacing & $20 \mu \mathrm{m}$
\end{tabular}




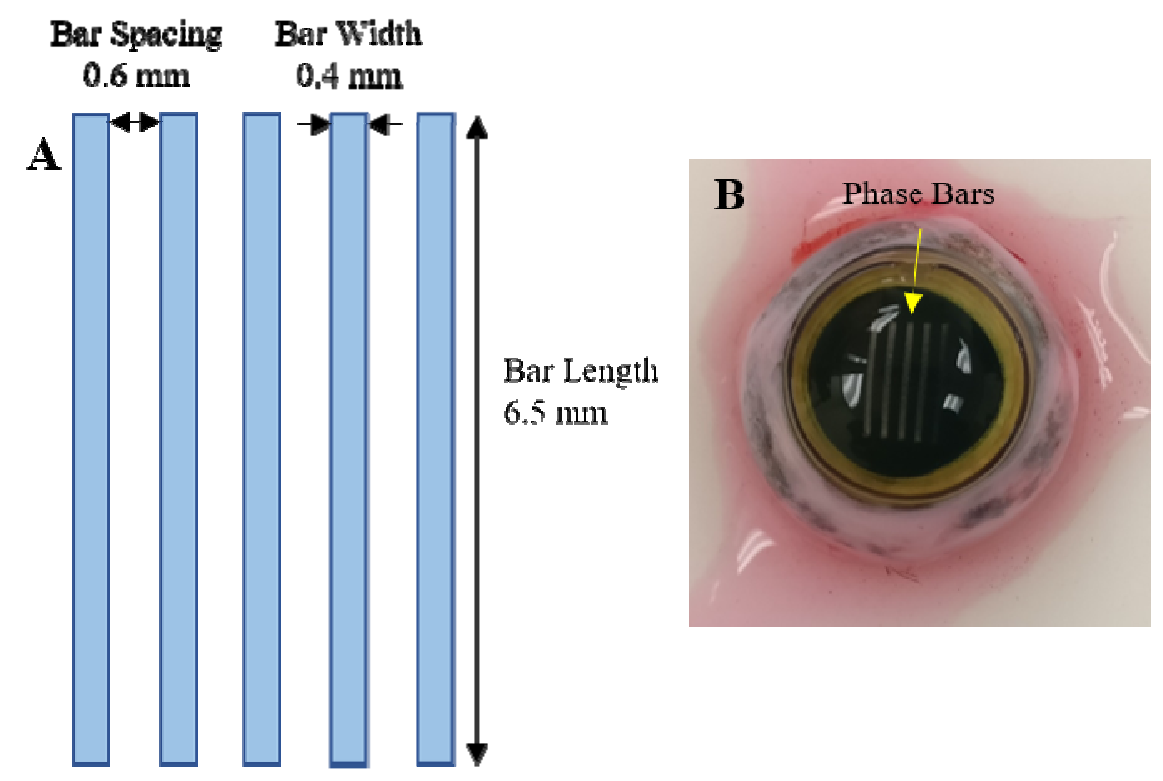

Figure 3: A) Diagram of the phase change calibration bars used for calibrating the bulk phase change with respect to delivered laser power in cornea. B) Picture of phase bars in an enucleated cat ocular globe immediately after IRIS calibration procedure. The bars were visible due to microbubbles, which dissipated over the following hour, leaving the cornea completely transparent again.

Immediately after writing, many of the higher laser power calibration bars showed microbubbles. Once the calibration writing was finished, the eyeballs were submersed in Optisol-GS and placed in the refrigerator for approximately an hour. This allowed the bubbles to dissipate, at which point the bars became completely transparent. After the bubbles dissipated, the eyeball was bisected approximately halfway between the cornea and the optic nerve. The retina, vitreous humor, aqueous humor, lens, and iris were removed, leaving only the cornea and approximately 4-8 $\mathrm{mm}$ of sclera surrounding the cornea. The cornea and surrounding sclera were then mounted into a custom wet cell. The wet cell was designed to fully submerse the cornea in fluid, either Optisol-GS or a saline solution, while the surrounding sclera was clamped to hold the cornea in place. Pressure could then be applied to the posterior surface of the cornea to mimic intraocular pressure (IOP), either through a tube attached to a syringe or simply by the hydrostatic pressure caused by elevating the tube to create a column of fluid. This put the cornea into its natural position, as in the eye, and smoothed the wrinkles that resulted when there was no additional pressure applied. Windows were placed on the front and rear to allow for transmissive measurement with a MZI. A cartoon of the dissection and wet cell mounting, as well as an exploded view of the actual wet cell design from SolidWorks, are shown in Fig. 4. 


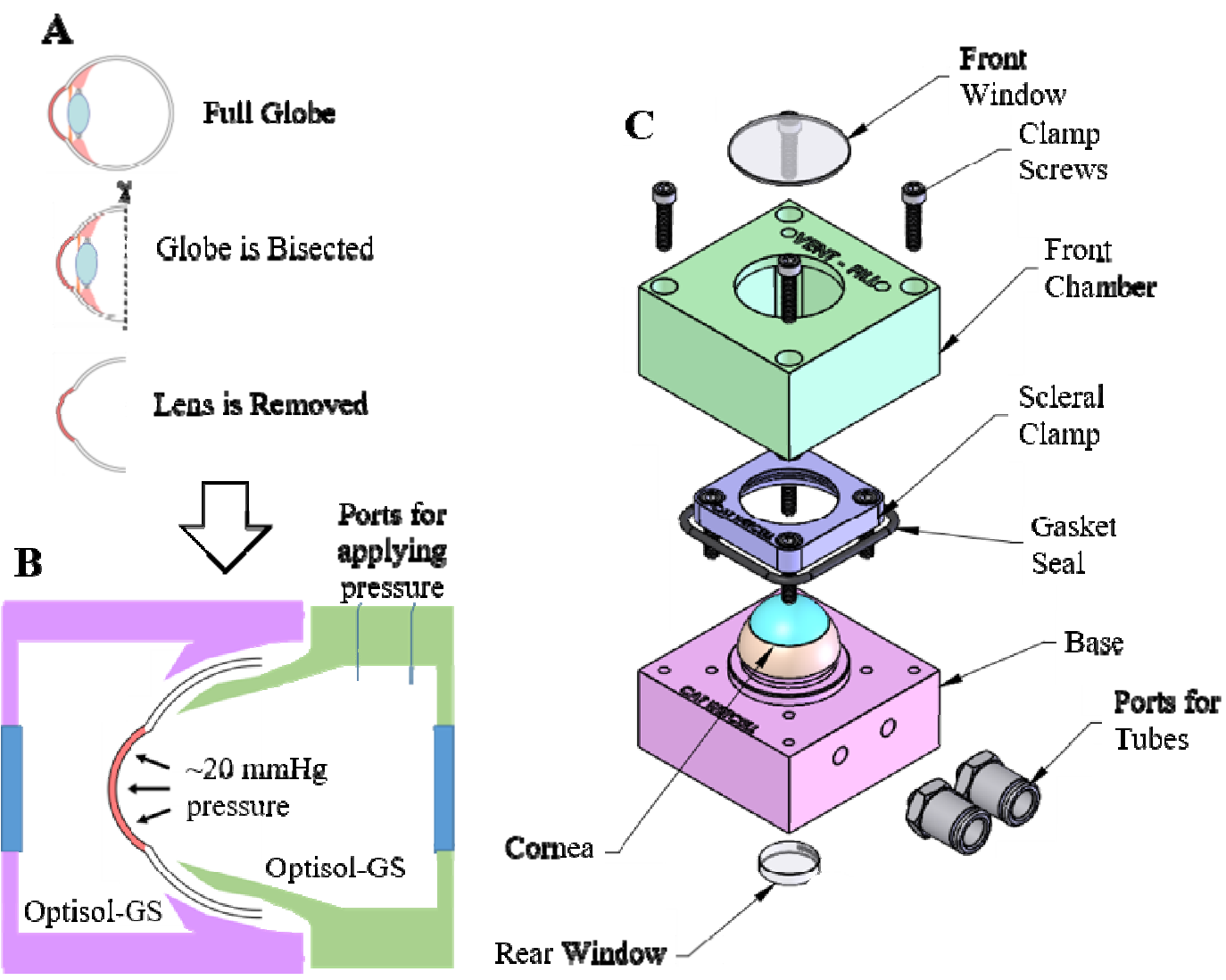

Figure 4: A) A cartoon showing the dissection of the eye where the globe is bisected and the lens is removed. The remaining cornea and sclera is then mounted into a wet cell, with a cartoon of the cross-section of this shown in the bottom left B). C) An exploded view taken from SolidWorks of the actual wet cell design showing how it was assembled.

\subsection{Phase Calibration Measurement in Excised Cornea}

With the cornea mounted in a wet cell, transmissive measurement of the induced bulk optical phase change could be performed using the MZI (Fig. 5A). The laser source used in the MZI measurements was a Helium-Neon (HeNe) laser operating at $632.8 \mathrm{~nm}$. The wet cell-mounted cornea was placed on a platform attached to a manual XYZ translation stage in the measurement arm of the MZI. Multiple interferograms and collocated bright field images were recorded of each IRIS phase bar (Fig. 5B). The bright field images were recorded by simply blocking the reference arm. 


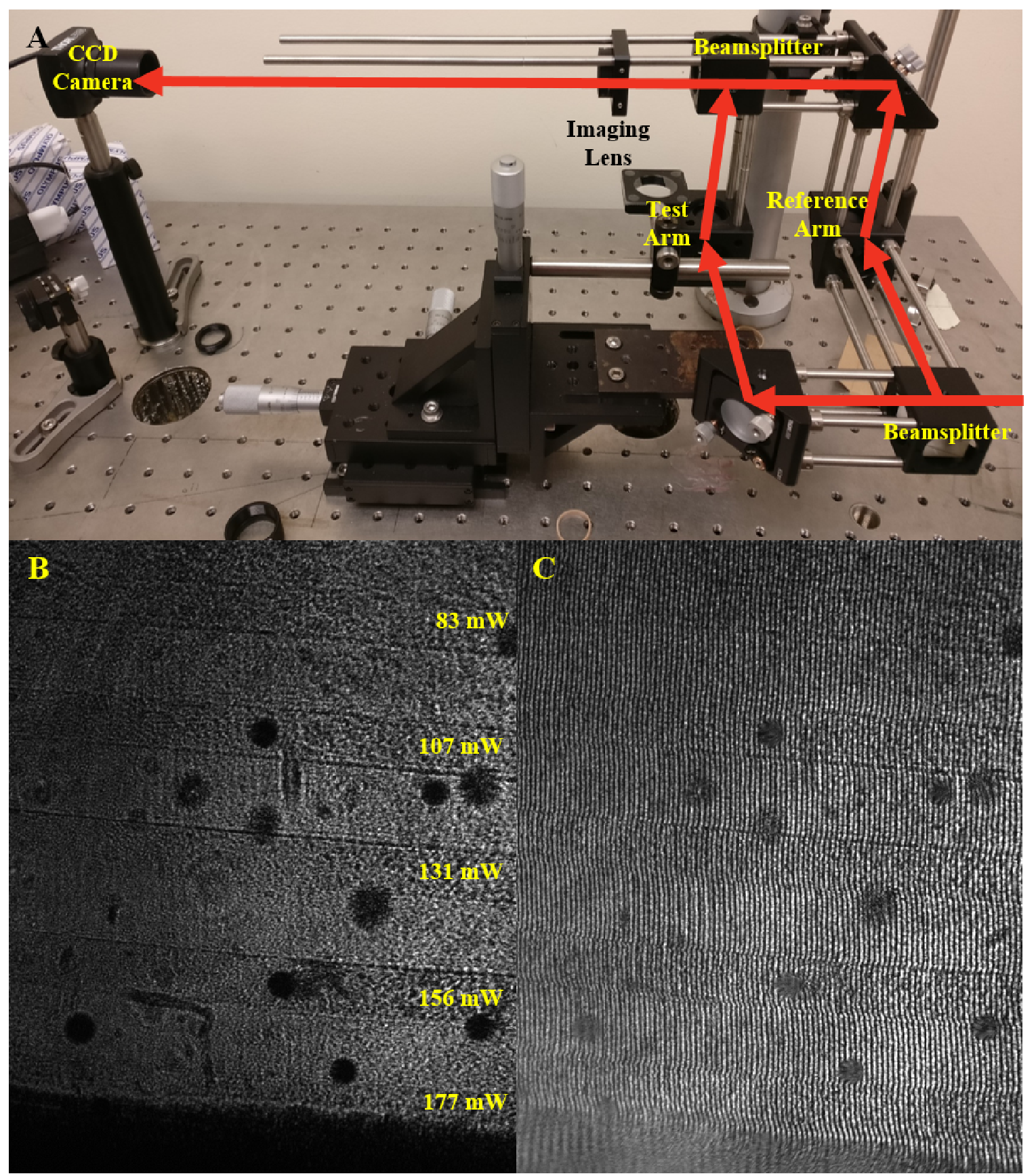

Figure 5: A) Mach-Zehnder interferomter used for measuring phase change induced in excised cornea. Measurement wavelength was $632.8 \mathrm{~nm}$. B) Bright field and C) interferogram of an example cornea mounted in the wet cell taken with the MZI. The laser power delivered for each bar is given. The black spots are bubbles and residual debris from dissection floating the surrounding fluid.

Once the interferograms were taken, they were processed into phase maps using a Fourier transform based algorithm (Fig. 6), as described previously. ${ }^{17}$ To calculate the phase change in each bar, small neighboring regions, one inside the bar and one outside the bar were averaged and the difference between the two was calculated. The regions taken had to be small and near each other due to low-frequency variation of the phase across the interferogram caused by residual aberrations and wrinkles in the cornea. To get an accurate assessment of the phase change induced at a given laser power, multiple pairs of small, neighboring regions were taken (Fig. 6). 
A

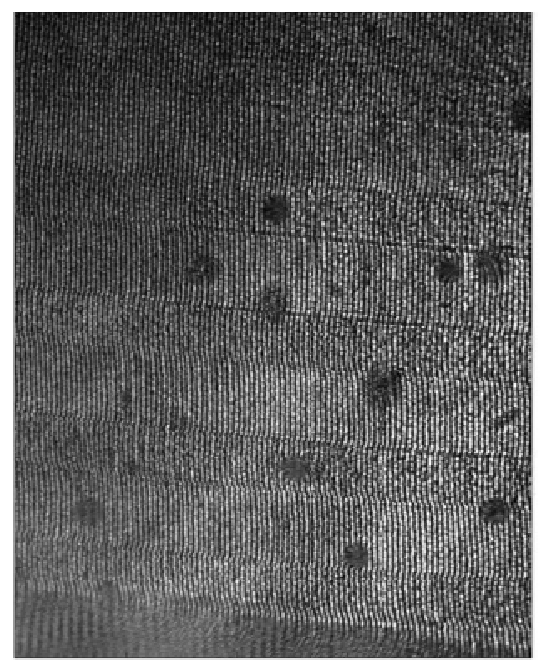

B

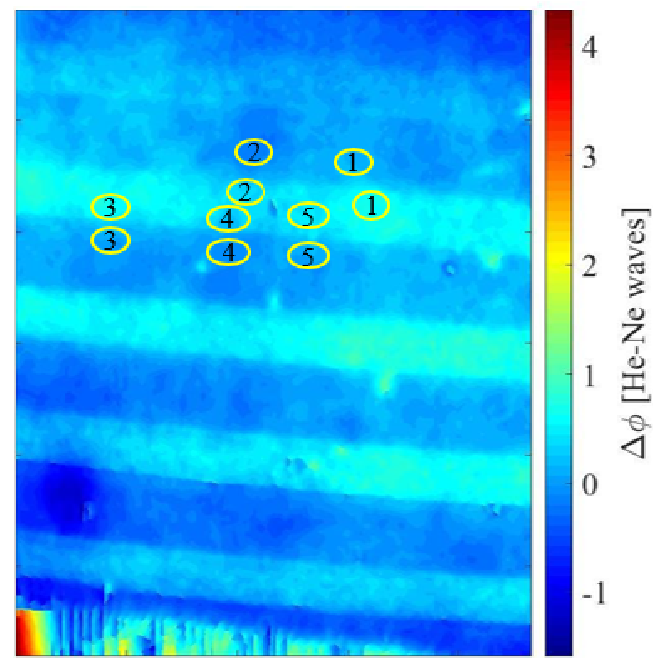

Figure 6: Example interferogram (A) and the corresponding phase map (B). Examples of sets of neighboring pairs of regions that could be taken for calculating the resultant phase change is shown with the yellow ellipses on the phase map. Each region has a number that denotes which pair it belongs to. Regions are taken along wrinkles or other low frequency variations to prevent those variations from affecting the calculated phase differences.

After computing the difference between multiple pairs of neighboring, background regions, results collected from inside individual bars were averaged to find the induced phase change at that power. This was done in two eyes from two different cats (Fig. 7). There seemed to be a saturation of the measured data above $160 \mathrm{~mW}$ (data not shown), but it is unknown as of the writing of this manuscript whether that saturation was physical or if it was a result of using enucleated globes. A linear fit was found for the truncated data and was used as the calibration curve for the design of the prescription for the in vivo creation of refractive structures in live cats.

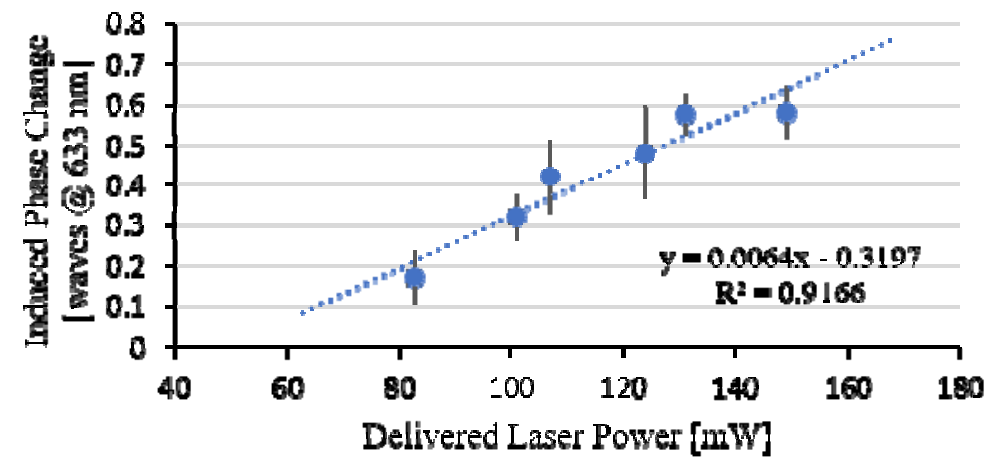

Figure 7: Plot of the induced bulk phase change in enucleated cat eyes as a function of the delivered laser power at the parameters given earlier in Table 1. Each point corresponds to the average of approximately 10 different pairs of regions from a single bar, as shown in Fig. 8, and the standard deviations were found from the standard deviation of that average. The data was truncated at $160 \mathrm{~mW}$ and a linear fit for the remaining data is shown. Each point corresponds to three layers of induced phase change. 


\section{DESIGN OF IRIS REFRACTIVE CORRECTORS FOR CORNEAL APPLICATIONS}

After computing the calibration curve for IRIS in cornea, the next step toward writing a full structure in vivo was to design the specific lens or refractive corrector that would be inscribed. The most fundamental way to describe a lens is through the accumulated optical phase of a wavefront. The thickness of the region of RI change using IRIS has never been accurately measured, but the nonlinear nature of the process would suggest that the RI change thickness was less than $10 \mu \mathrm{m}$. This was thin enough that the IRIS structure could be reasonably treated as a thin lens, which in turn, can be treated as a phase transformation represented by

$$
t_{l}(r)=e^{-i \frac{k r^{2}}{2 f}}
$$

where $k$ is the wavenumber in freespace, $r$ is the radial position in the lens, and $f$ is the focal length of the lens. ${ }^{18}$ The phase change profile to create a lens using the calibration from the previous section is therefore

$$
\Delta \varphi(r)=\frac{k r^{2}}{2 f} .
$$

This phase is in units of radians. By converting to variables that are more widely recognizable in the ophthalmological field and converting the units to waves at a design wavelength, we find

$$
\Delta \varphi(r)=\frac{D r^{2}}{2 \lambda},
$$

where $D$ is the optical power of the lens in Diopters and $\lambda$ is the design wavelength. For clinical relevance, the power of the lens must have a magnitude of at least $1.5 \mathrm{D}(f=666.7 \mathrm{~mm})$. Using equation (3) with a design wavelength of $633 \mathrm{~nm}$, a $1.5 \mathrm{D}$ lens over the clinically relevant optical zone of $6.0 \mathrm{~mm}$ yields a maximum phase change of 10.66 waves at $633 \mathrm{~nm}$. This is significantly higher than the IRIS process can achieve in cornea.

While the total magnitude of the phase difference over the full structure is much too great for IRIS, it is possible to wrap the phase at 1 wave intervals. This would create a structure with a 1 wave maximum phase difference across the structure, while maintaining the same optical power. This type of structure is called a Fresnel lens, also known as a kinoform lens. ${ }^{19-21}$ By collapsing the phase in this way, a structure with a series of zones with phase wrapping between 0 and 1 wave is achieved. A comparison of the two different structures can be seen in Fig. 8.

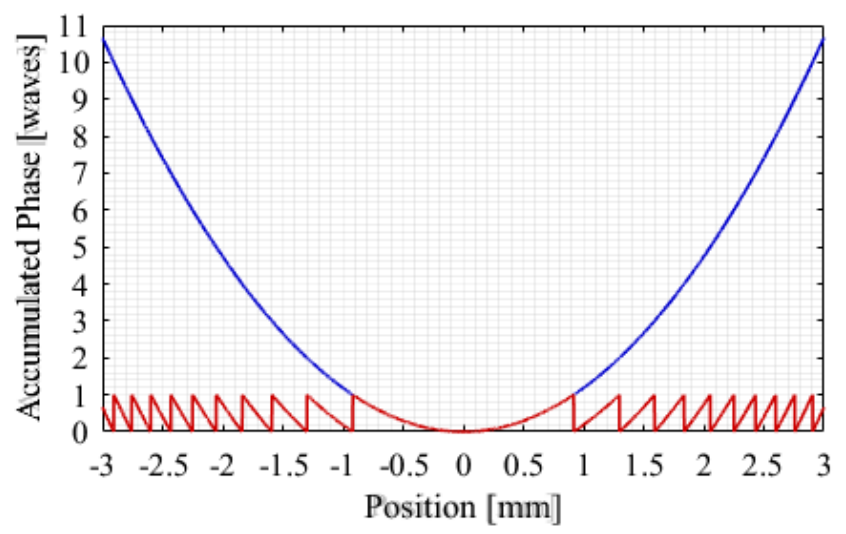

Figure 8: Comparison of a cross-sections of the required accumulated phase for a $-1.5 \mathrm{D}$ lens across a 6 mm diameter region with a standard phase profile (blue) and a Fresnel lens type phase profile (red). Accumulated phase was simulated at $633 \mathrm{~nm}$.

The phase Fresnel lens works as a diffractive optical element (DOE). With DOEs, the focus is dependent on the number of zones, with more tightly spaced zones resulting in a more powerful lens. This is beneficial for writing a lens using IRIS because, while the optical phase change induced was characterized, the uncertainty in the calibration was 
relatively large. However, if the peak phase change is not equal to an integer number of waves, the diffraction is not perfectly matched and the light will be directed into different diffractive orders, corresponding to different foci along the optical axis. These foci are located at integer multiples of the first order focus location. This creates what is called a multi-focal optic, which has been leveraged for design of IOLs for people with presbyopia. ${ }^{22}$ The amount of light sent into the different orders, or diffraction efficiency, can be calculated using

$$
\eta(m, \mu)=\operatorname{sinc}^{2}\left[\pi\left(m-m_{0} \mu\right)\right]
$$

where $m$ is the diffracted order of interest, $m_{0}$ is the design order, and $\mu$ is a parameter determined by the departure from the design zone height. In the case of the structures used in this design, $\mu$ can be calculated with

$$
\mu=\left(\frac{h}{h_{0}}\right)\left(\frac{\lambda_{0}}{\lambda}\right)
$$

where $h$ is the peak-to-valley phase height of the structure, $h_{0}$ is the design phase height necessary to diffract all light to the design order, $\lambda_{0}$ is the design wavelength, and $\lambda$ is the measurement or observation wavelength. With $\mu=1, \eta$ is 1 for the design order and 0 for all other orders.

The Fresnel phase profile shown in red in Figure 838 was converted to an incident laser power using the fit equation from Fig. 7. This was used as the laser power prescription in one dimension for the design of the IRIS structure. The structure was designed as a cylindrical Fresnel lens, with the prescription governing the laser power delivered along the $\mathrm{x}$ - (linear stage-) axis, and the laser power remaining constant along the $\mathrm{y}$ - (flexure stage-) axis. The software was developed such that an AOM voltage to laser power calibration could be loaded onto the computer on the day of writing, and the software would convert the laser power at a position into an AOM voltage in real time.

\section{REFRACTIVE CORRECTIONS IN LIVE CATS}

\subsection{In vivo IRIS Procedure}

Two eyes, one each from two different young adult domestic short hair cats were used for in vivo IRIS experiments. In the months leading up to the IRIS procedure, the cats were behaviorally trained to fixate along the optical axis of a custom Shack-Hartmann wavefront sensor (SHWS), as previously described. ${ }^{23,24}$ Prior to the IRIS procedure, several spot array patterns were collected using the SHWS to construct a baseline wavefront for the eye that was to undergo IRIS, so that its wavefronts post-IRIS could be compared.

For the IRIS procedure, the cats were put under surgical (ketamine, $5 \mathrm{mg} / \mathrm{kg}$, dexmedetomidine hydrochloride $0.04 \mathrm{mg} / \mathrm{kg}$ ) and topical anesthesia (proparacaine $0.5 \%$; Falcon). The cat's head was then stabilized using a titanium headpost attached to the skull. The headpost was attached with a dowel pin to a 3-axis manual stage with the attachment point itself designed to provide rotation about the axis of the headpost. The cornea was applanated using the applanator shown in Figure 2. The parameters for writing the IRIS Fresnel structures were the same as those listed in Table 1, except for bar size parameters, which were not applicable. Each Fresnel lens was designed to be $5.9 \mathrm{~mm}$ in diameter, following the phase profile for a $-1.5 \mathrm{D}$ Fresnel lens shown in Fig. 8. The flexure based scanning system operated over a $6.5 \mathrm{~mm}$ by $6.0 \mathrm{~mm}$ region with all points outside the $5.9 \mathrm{~mm}$ circular Fresnel lens set to zero laser power through the AOM. Before mounting the cat, an AOM voltage to delivered laser power calibration curve was measured. This curve was used to create the AOM voltage prescription corresponding to the Fresnel structure shown in Fig. 8. With the cat mounted and the prescription calculated, the IRIS procedure was performed with a total runtime for all three layers of 30 minutes.

Once the IRIS procedure was complete, the applanator was removed from the eye and the cat was unmounted from the system. Once the cats recuperated enough from anesthesia to cooperate with wavefront measurements (usually 1-2 days), each eye's wavefront was measured using the SHWS at regular intervals over the following 6 months. As of the writing of this manuscript, wavefronts continue to be measured in these two eyes. 
For this work, Zernike Polynomials were used to describe the reconstructed wavefront, in accordance with standards for reporting ophthalmological aberrations. ${ }^{25}$ The Zernike coefficients will be referred to as $C_{j}$ where $j$ represents the index of a specific Zernike coefficient. The most important Zernike terms for ophthalmological aberrations are $C_{4}$, representing defocus, and $C_{3}$ and $C_{5}$, representing the two astigmatism terms. The relations governing the conversion from these Zernike coefficients to the corresponding optical powers are

$$
\begin{gathered}
D E F=\frac{4 \sqrt{3} C_{4}}{r^{2}}, \\
C Y L=-\frac{4 \sqrt{6} \sqrt{\left(C_{3}\right)^{2}+\left(C_{5}\right)^{2}}}{r^{2}}, \text { and } \\
\varphi=\frac{1}{2} \tan ^{-1}\left(\frac{C_{3}}{C_{4}}\right)
\end{gathered}
$$

where $D E F$ is the paraxial wavefront defocus, $C Y L$ is the paraxial wavefront cylinder, $\phi$ is the angle of the cylinder axis, and $r$ is the radius of the measurement zone. These equations are of opposite power from the traditional ophthalmological representation because it is the second-order optical power, not the spectacle correction, that is of interest in this work ${ }^{26,27}$. In addition to the optical powers, it is often of interest to quantify the overall higher-order aberrations. This is often represented with the root mean square (RMS):

$$
R M S=\sqrt{\sum_{j}\left(C_{j}\right)^{2}} .
$$

To quantify the higher-order aberrations, the sum in Eq. 9 is taken from $j=6$ to 65 and is call the higher-order root mean square (HORMS) ${ }^{27}$.

\subsection{In vivo IRIS results}

Immediately after the IRIS procedure was performed, pictures were taken of each eye (Fig. 9). Microbubbles were visible in the cornea immediately after the procedure, but dissipated over the following 30-60 minutes, leaving the cornea completely transparent. In the case of Fresnel lenses, these bubbles made the phase-wrapped structure immediately apparent, as they only formed in the higher phase change regions of the lens (where the most laser power was delivered). 


\section{$\mathbf{A}$}

B

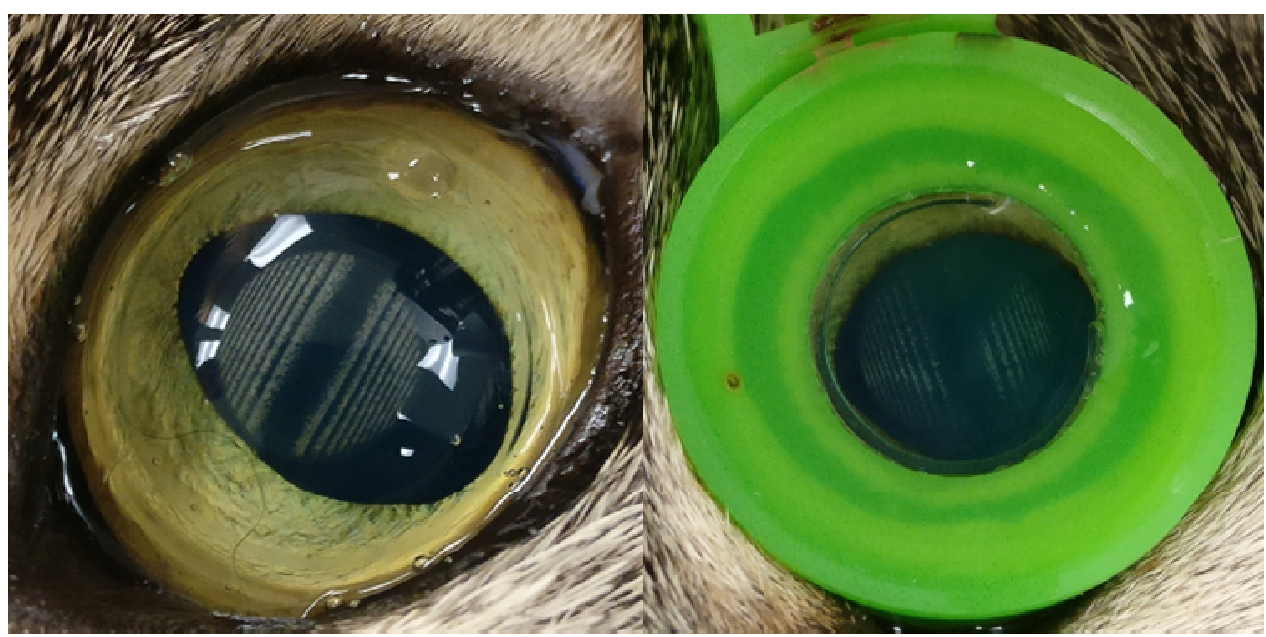

Figure 9: Pictures of cylindrical Fresnel lenses written with IRIS, in vivo, into two different cats. The visible areas are microbubbles formed in the higher laser power regions corresponding to higher induced phase change. These bubbles dissipated over the following 30-60 minutes, as with the enucleated eyes. The green device in B) is the applanator.

Over the following six months, the cylindrical power calculated from the SHWS wavefront data remained stable for both eyes; although the magnitude of induced cylinder differed between the two eyes. The average cylindrical power change from baseline was $-1.40 \pm 0.17 \mathrm{D}$ for one cat and $-0.96 \pm 0.10 \mathrm{D}$ for the second cat. The defocus change from baseline centered around approximately $-0.25 \mathrm{D}$ for both eyes. The data are summarized in Table 2 and plotted in Fig. 10 .

Table 2: Summary of measured Zernike coefficients and calculated refractive powers

\begin{tabular}{lcc}
\multicolumn{1}{c}{ Parameter } & Cat 1 OD & Cat 2 OD \\
\hline$\Delta$ Zernike Coefficients $[\mu \mathrm{m}]$ & & \\
$\Delta \mathrm{C} 3$, astigmatism at $45^{\circ}$ & $-0.41 \pm 0.07$ & $-0.15 \pm 0.10$ \\
$\Delta \mathrm{C} 4$, defocus & $-0.26 \pm 0.21$ & $-0.22 \pm 0.22$ \\
$\Delta \mathrm{C} 5$, astigmatism at $0^{\circ}$ & $-0.79 \pm 0.11$ & $-0.76 \pm 0.27$ \\
& & \\
$\Delta$ HORMS $[\mu \mathrm{m}]$ & $0.50 \pm 0.10$ & $0.37 \pm 0.04$ \\
& & \\
$\begin{array}{l}\Delta \text { Refractive Power }[\mathrm{D}] \\
\Delta \text { Defocus }\end{array}$ & $-0.29 \pm 0.23$ & $-0.24 \pm 0.24$ \\
$\Delta$ Cylinder & $-1.40 \pm 0.17$ & $-0.96 \pm 0.10$
\end{tabular}



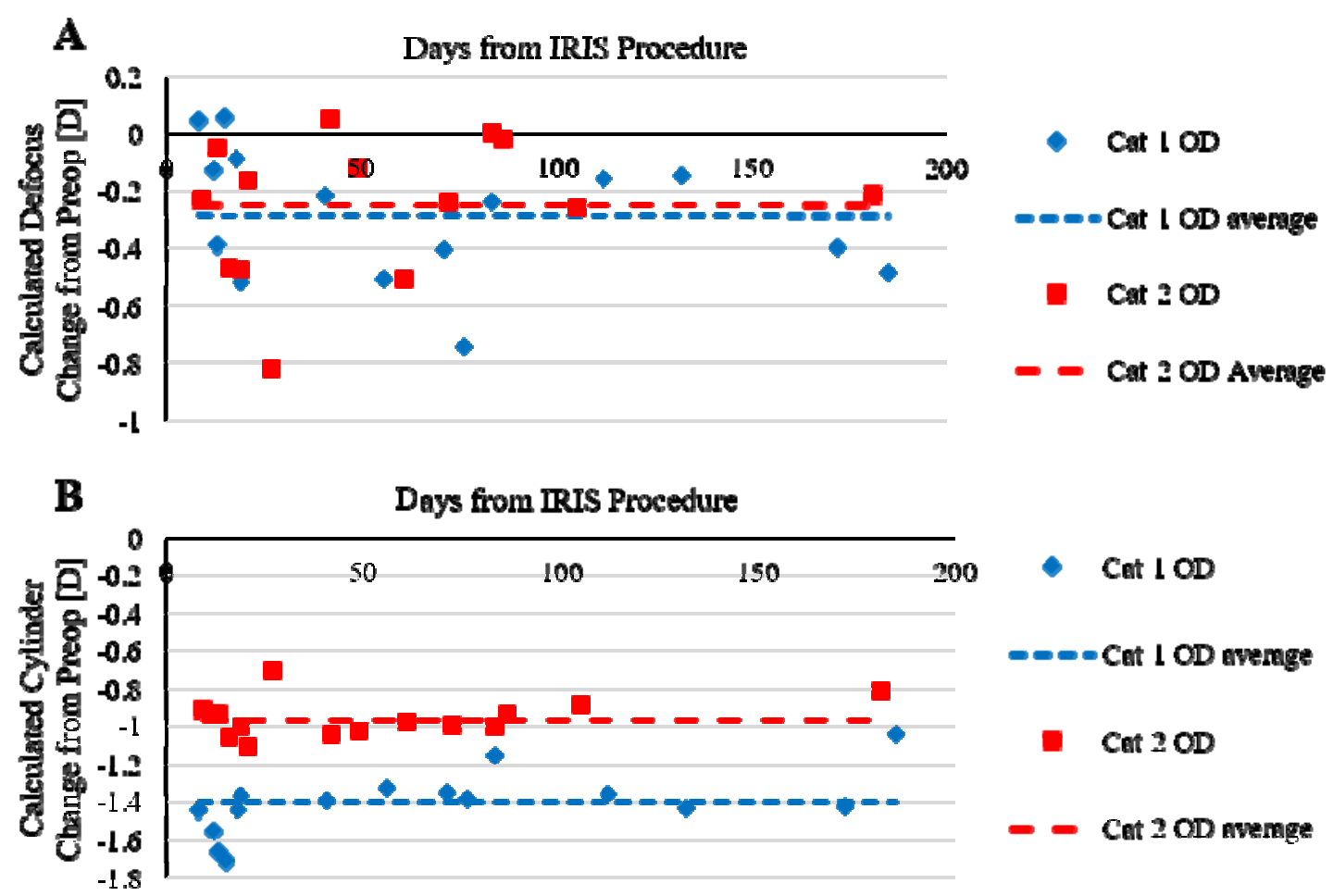

Figure 10: Plots of the change from preoperative wavefronts for defocus (A) and cylinder (B) power.

\subsection{Discussion}

Overall, the present experiments represent a significant technological and practical advance over previous in vivo results with IRIS. ${ }^{16}$ The development of new scanning modalities in the custom flexure stage, ${ }^{15}$ as well as advances in metrology, have significantly improved our ability to study the IRIS process and its refractive performance in-vivo. The measured dioptric power change of both cylinder and defocus proved to be more stable over time than previously obtained. Also, the defocus induced in each eye was consistent between eyes, demonstrating a more repeatable process and measurements. However, the HORMS was higher for these eyes than the eyes written on the previous system ${ }^{16}$, which is consistent with the larger measurement zone and the significantly more complex Fresnel structure used here. Importantly, the present results demonstrate - for the first time - feasibility of fully arbitrary phase structures over clinically relevant optical zones using the IRIS approach. This was embodied in the complexity of the Fresnel cylinder lenses successfully written in vivo.

While refractive change was observed in both written eyes, cat 2 displayed less change and did not meet our design goal of -1.5 D. A distinct visible difference can be seen in Fig. 9 between the two eyes. However, the operations on each eye seemed to go smoothly, with no obvious problems. It is not known at this time what caused the difference in induced wavefront change between the two eyes. More study will be needed to assess the inter-eye variability of IRIS to determine if this is due to variability in the system or an existing issue with the eye of that cat.

The present study thus represents a crucial step in the process of achieving refractive correctors in human eyes with IRIS. In parallel with this work, Wozniak et al. showed that comparable refractive index change is possible in humans and cat corneas. ${ }^{28}$ Another important step toward clinical application is to demonstrate safety of the IRIS procedure. Published results using our previous system showed that IRIS causes less cell death and DNA disruption to the cornea than LASIK when performed in enucleated cat globes. ${ }^{29}$ Although results of such work are outside the scope of the present experiments, ongoing studies are now examining the safety of our improved IRIS protocol and Fresnel lenses inscribed over a larger, $5 \mathrm{~mm}$ diameter optical zone. 


\section{CONCLUSION}

The present results present a new process for creating in vivo refractive corrections using IRIS, from system calibration to structure design, to the final in vivo procedure and metrology. A calibration between phase change and delivered laser power was attained in the corneas of enucleated cat eyes measured in a wet cell with an MZI. This calibration showed a mostly linear trend up to a saturation point between 0.6 and 0.8 waves of induced phase change at $633 \mathrm{~nm}$. This calibration was used to design a $-1.5 \mathrm{D}$ refractive corrector in the form of a Fresnel lens. Finally, using the IRIS process, the designed Fresnel lens was inscribed into two eyes of two living cats, producing final refractive corrections that could be measured in the awake, behaving state. The average attained refractive change was $-1.40 \pm 0.17 \mathrm{D}$ in one eye and $0.96 \pm 0.10 \mathrm{D}$ in the second eye, with very little induced defocus. These corrections were measured over the course of six months with a SHWS and were stable over that time.

\section{ACKNOWLEDGEMENTS}

The authors would like to thank Margaret DeMagistris and Christine Callan for their extensive help in handing the animals and performing the wavefront measurements in vivo. We also thank Gustavo Gandara Montano for developing the MZI analysis software used in this work and Dr. Len Zheleznyak for constructing the original version of the SHWS used in cats.

This project was supported by an unrestricted grant to the University of Rochester's Department of Ophthalmology from the Research to Prevent Blindness (RPB) Foundation, by the National Institutes of Health (R01 grant EY015836 to KRH; Core grant P30 EY01319 to the Center for Visual Science); by the National Science Foundation (grants IIP: 1549700 and IIP: 1738506), by a grant from Clerio Vision, Inc. with matching funds from the University of Rochester's Center for Emerging \& Innovative Sciences, a NYSTAR-designated Center for Advanced Technology, and by an Incubator Grant from the University of Rochester's CTSI Scientific Advisory Committee (SAC).

The authors declare the following interests: W. H. Knox, K.R. Huxlin, and J. D. Ellis have founder's equity in Clerio Vision, Inc., which partially supported this research. W. H. Knox, K. R. Huxlin, and J. D. Ellis have no fiduciary or management responsibility in Clerio Vision, Inc.

\section{REFERENCES}

[1] S. L. Trokel, R. Srinivasan, and B. Braren, "Excimer laser surgery of the cornea," American journal of ophthalmology, 96(6), 710-715 (1983).

[2] R. Krueger, S. Trokel, and H. Schubert, "Interaction of ultraviolet laser light with the cornea," Investigative ophthalmology \& visual science, 26(11), 1455-1464 (1985).

[3] C. R. Munnerlyn, S. J. Koons, and J. Marshall, "Photorefractive keratectomy: a technique for laser refractive surgery," Journal of Cataract \& Refractive Surgery, 14(1), 46-52 (1988).

[4] L. G. Pallikaris, M. E. Papatzanaki, E. Z. Stathi et al., "Laser in situ keratomileusis," Lasers in surgery and medicine, 10(5), 463-468 (1990).

[5] R. Ambrósio Jr, T. Tervo, and S. E. Wilson, "LASIK-associated dry eye and neurotrophic epitheliopathy: pathophysiology and strategies for prevention and treatment," Journal of refractive surgery, 24(4), 396-407 (2008).

[6] R. R. Mohan, A. E. Hutcheon, R. Choi et al., "Apoptosis, necrosis, proliferation, and myofibroblast generation in the stroma following LASIK and PRK," Experimental eye research, 76(1), 71-87 (2003).

[7] L. Ding, R. Blackwell, J. F. Kunzler et al., "Large refractive index change in silicone-based and non-siliconebased hydrogel polymers induced by femtosecond laser micro-machining," Optics express, 14(24), 1190111909 (2006).

[8] L. Ding, D. Jani, J. Linhardt et al., "Large enhancement of femtosecond laser micromachining speed in dyedoped hydrogel polymers," Optics express, 16(26), 21914-21921 (2008). 
[9] L. Xu, and W. H. Knox, "Lateral gradient index microlenses written in ophthalmic hydrogel polymers by femtosecond laser micromachining," Optical Materials Express, 1(8), 1416-1424 (2011).

[10] L. Ding, W. H. Knox, J. Bühren et al., "Intra-tissue Refractive Index Shaping (IRIS) of the cornea and lens using a low-pulse-energy femtosecond laser oscillator," Investigative ophthalmology \& visual science, 49(12), $5332(2008)$.

[11] L. Ding, [Micro-processing of polymers and biological materials using high repetition rate femtosecond laser pulses] University of Rochester, (2009).

[12] L. J. Nagy, L. Ding, L. Xu et al., "Potentiation of femtosecond laser intratissue refractive index shaping (IRIS) in the living cornea with sodium fluorescein," Investigative ophthalmology \& visual science, 51(2), 850-856 (2010).

[13] L. Xu, [Femtosecond laser processing of ophthalmic materials and ocular tissues: a novel approach for noninvasive vision correction] University of Rochester, (2013).

[14] L. Xu, W. H. Knox, M. DeMagistris et al., "Noninvasive intratissue refractive index shaping (IRIS) of the cornea with blue femtosecond laser light," Investigative ophthalmology \& visual science, 52(11), 8148-8155 (2011).

[15] D. R. Brooks, N. S. Brown, D. E. Savage et al., "Precision large field scanning system for high numerical aperture lenses and application to femtosecond micromachining of ophthalmic materials," Review of Scientific Instruments, 85(6), 065107 (2014).

[16] D. E. Savage, D. R. Brooks, M. DeMagistris et al., "First demonstration of ocular refractive change using blueiris in live cats," Investigative ophthalmology \& visual science, 55(7), 4603 (2014).

[17] G. A. Gandara-Montano, A. Ivansky, D. E. Savage et al., "Femtosecond laser writing of freeform gradient index microlenses in hydrogel-based contact lenses," Optical Materials Express, 5(10), 2257-2271 (2015).

[18] J. W. Goodman, [Introduction to Fourier optics] Roberts and Company Publishers, (2005).

[19] K. Miyamoto, "The phase Fresnel lens," JOSA, 51(1), 17-20 (1961).

[20] J. Jordan, P. Hirsch, L. Lesem et al., "Kinoform lenses," Applied Optics, 9(8), 1883-1887 (1970).

[21] D. C. O'Shea, T. J. Suleski, A. D. Kathman et al., "Diffractive optics: design, fabrication, and test," (2004).

[22] V. Portney, "Light distribution in diffractive multifocal optics and its optimization," Journal of Cataract \& Refractive Surgery, 37(11), 2053-2059 (2011).

[23] K. R. Huxlin, G. Yoon, L. Nagy et al., "Monochromatic ocular wavefront aberrations in the awake-behaving cat," Vision Research, 44(18), 2159-2169 (2004).

[24] L. J. Nagy, S. MacRae, G. Yoon et al., "Photorefractive keratectomy in the cat eye: Biological and optical outcomes," Journal of Cataract \& Refractive Surgery, 33(6), 1051-1064 (2007).

[25] L. N. Thibos, R. A. Applegate, J. T. Schwiegerling et al., "Standards for reporting the optical aberrations of eyes," Journal of refractive surgery, 18(5), S652-S660 (2002).

[26] L. N. Thibos, X. Hong, A. Bradley et al., "Accuracy and precision of objective refraction from wavefront aberrations," Journal of vision, 4(4), 9-9 (2004).

[27] A. Guirao, and D. R. Williams, "A method to predict refractive errors from wave aberration data," Optometry \& Vision Science, 80(1), 36-42 (2003).

[28] K. T. Wozniak, S. M. Gearhart, D. E. Savage et al., [Comparable Change in Stromal Refractive Index of Cat and Human Corneas Following Blue-IRIS], Journal of Biomedical Optics(2017).

[29] K. T. Wozniak, N. Elkins, D. R. Brooks et al., "contrasting cellular damage after Blue-iris and Femto-lasik in cat cornea," Experimental eye research, 165, 20-28 (2017). 